\title{
Clinics in diagnostic imaging (168)
}

Yusheng Keefe Lai ${ }^{1}$, MBBs, Rameysh Danovani Mahmood ${ }^{1}$, MB BCh BAO, FRCR

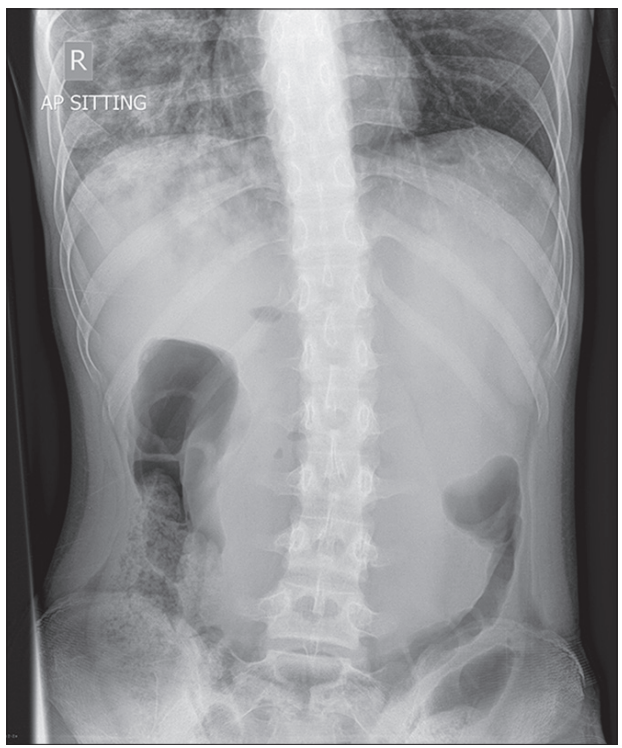

Fig. 1 Erect abdominal radiograph.
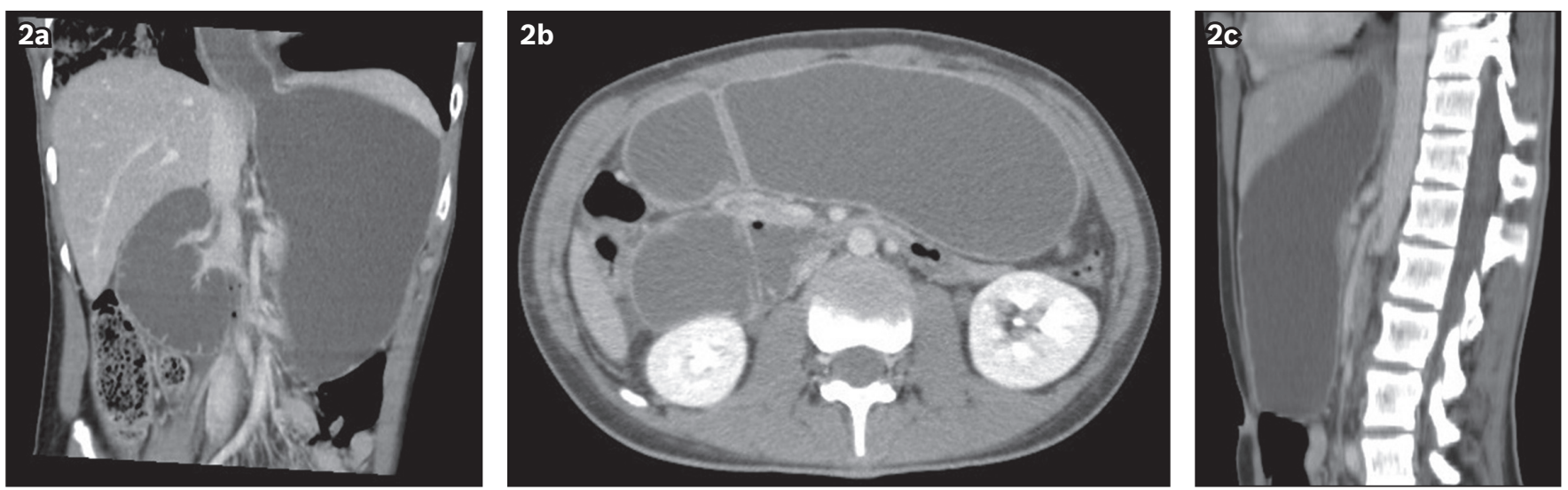

Fig. 2 (a) Coronal oblique; (b) axial; and (c) sagittal CT reconstructions of the abdomen.

\section{CASE PRESENTATION}

A 16-year-old Chinese male patient, who had a past medical history of hyperventilation complicated by carpopedal spasms, presented to the emergency department with constipation of five days' duration, colicky abdominal pain, lethargy, weakness and body aches. He was still able to pass flatus, and did not complain of vomiting, fever, chills or rigors. On examination, the abdomen was soft and non-tender. Digital rectal examination was unremarkable, as was the full blood count, except for a raised total white blood cell count of $16.6 \times 10^{\%} / \mathrm{L}$. Renal and liver function tests and urinalysis were also unremarkable. The patient was hospitalised for further investigation of constipation. During his hospital stay, abdominal radiography was performed (Fig. 1). He subsequently desaturated acutely, and computed tomography (CT) was performed (Fig. 2). What do the images show? What is the diagnosis? 


\section{IMAGE INTERPRETATION}

Erect abdominal radiograph (Fig. 1) shows mass effect displacing the transverse colon inferiorly. The mass appears to arise from the expected location of the stomach. CT images (Fig. 2) show a large, distended oesophagus, stomach and duodenum up to its third portion. No obvious mural or extrinsic duodenal mass lesion is visualised. At the transition point, the superior mesenteric artery (SMA) and aorta show a narrow aortomesenteric angle and a short aortomesenteric distance. There is also a consolidative change noted in the visualised right lung base (dedicated CT thorax was performed, but images are not shown).

\section{DIAGNOSIS}

Superior mesenteric artery syndrome (SMAS) complicated by aspiration pneumonia.

\section{CLINICAL COURSE}

The patient was admitted to the intensive care unit, where a nasogastric tube was inserted. Overnight, 2,170 mL of fluid was aspirated via the tube. The tube was subsequently changed to a double lumen nasojejunal feeding tube under fluoroscopic guidance and a weight-gain plan was instituted under the supervision of a dietitian. He was also started on amoxicillin/ clavulanic acid (Augmentin ${ }^{\mathrm{TM}}$ ) for the treatment of aspiration pneumonia. The psychiatric team evaluated the patient for possible anxiety and referred him for relaxation therapy. He was subsequently followed up as an outpatient by the general surgery team, with a view to surgical intervention if conservative management was unsuccessful. A recent repeat CT showed no change to the narrow aortomesenteric angle and short aortomesenteric distance, although the duodenal obstruction had resolved. This configuration may predispose the patient to further episodes of SMAS.

\section{DISCUSSION}

SMAS was first described in 1861 as a rare cause of high intestinal obstruction, ${ }^{(1)}$ with a reported incidence of $0.013 \%-0.3 \%$ based on barium studies. ${ }^{(2)}$ The condition is secondary to compression of the third horizontal portion of the duodenum between the SMA and aorta.

There are a myriad of risk factors for SMAS. Decreased thickness of the retroperitoneal visceral fat pad with consequent narrowing of the aortomesenteric angle and distance can be seen in entities that cause rapid weight loss. These include conditions such as anorexia nervosa, gastric bypass surgery and severe injuries. ${ }^{(2,3)}$ Modulation of the angle can also be seen following surgical correction of scoliosis ${ }^{(4)}$ and during periods of rapid height gain. Intrinsic predisposing factors include a congenitally high insertion of the ligament of Treitz or a low origin of the SMA.(2) Another predisposed group of patients are those who have neurological impairments such as cerebral palsy, suggesting that weakening of the dynamic abdominal or spinal muscular brace is a contributing factor. ${ }^{(4)}$ Regardless of the original insult, SMAS is a self-reinforcing condition in which obstruction and poor oral intake result in further obstruction. ${ }^{(5)}$
Symptoms of SMAS include nausea, vomiting (either nonbilious or bilious), colicky abdominal pain, lethargy, weakness and weight loss. Complications include dehydration, electrolyte disturbances, aspiration pneumonia, failure to thrive, gastric ischaemia and perforation, and oesophageal rupture. ${ }^{(2,6)}$ Frequently, the symptoms are nonspecific, as in the present case. Symptom relief when assuming an anatomical position that widens the aortomesenteric distance (e.g. prone and left lateral decubitus positions) may suggest SMAS..$^{(2,3)}$

SMAS is difficult to diagnose due to its rarity and the presence of more common differentials such as malrotation with midgut volvulus, local obstructing mass, pancreatitis, or biliary or peptic ulcer disease. Therefore, both the referring clinician and radiologist need to maintain a high index of suspicion. We focus on some important differentials of SMAS in the rest of the discussion.

Malrotation in the adult population commonly represents the 'nonrotation' end of the spectrum of midgut rotation anomalies. In this discussion, malrotation refers only to 'nonrotation'. In malrotation, the bowel is abnormally positioned such that the large bowel lies to the left of the small bowel. Typically, the duodenaljejunal junction lies to the right of the spine, below the level of the pylorus. Importantly, there is concomitant narrowing of the small bowel mesenteric pedicle, which is a predisposing factor for midgut volvulus. Commonly associated with malrotation are abnormal fibrous Ladd's bands that run across the small bowel to attach to the ascending colon, which is a risk factor for internal hernias. Although a diagnosis of malrotation with midgut volvulus is often associated with the paediatric population (Fig. 3), it should be suspected when an adult presents with symptoms of small bowel obstruction, particularly when the transition point is located at the level of the duodenum. Plain radiography is neither sensitive nor specific for malrotation, although it may be suspected when there is predominance of large bowel gas in the left side of the colon and small bowel gas on the right side, as described in the pathologic anatomy above. On CT, besides the radiologic findings, the abnormal relationship of the SMA to the superior mesenteric vein (SMV) was also revealed; there can be a reversal of the normal right-left relationship of the SMV to the SMA, or the SMV can be more anterior to the SMA (Fig. 4). In midgut volvulus, the 'swirl-sign' of the mesentery wrapping around the SMA, with dilated stomach and small bowel loops, collapse of the colon, and engorgement of SMVs, can be seen. ${ }^{(7)}$

Local masses can also cause extrinsic compression on the duodenum with subsequent obstructive symptoms. A full exposition of the different kinds of masses in the region of the duodenum is beyond the scope of this paper, although one common entity is worth mentioning. Pancreatic pseudocysts develop approximately 4-6 weeks after an acute attack of pancreatitis. Their size varies, and they can resolve spontaneously. ${ }^{(8)}$ On cross-sectional imaging, they more commonly appear to be unilocular rather than multilocular. Also, they typically have thin, smooth walls or thick walls of uniform thickness and without solid components, wall calcification or central scarring. The presence of internal dependent debris is 


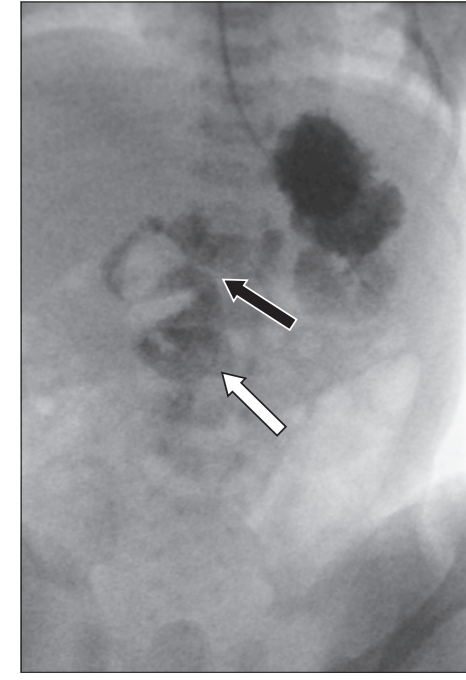

Fig. 3 Abdominal fluoroscopic image of a child with bilious emesis shows the duodenal-jejunal junction (black arrow) situated to the right of the spine and below the level of the pylorus, which is compatible with malrotation. Note the corkscrew appearance of the proximal jejunal loops (white arrow) in keeping with midgut volvulus.

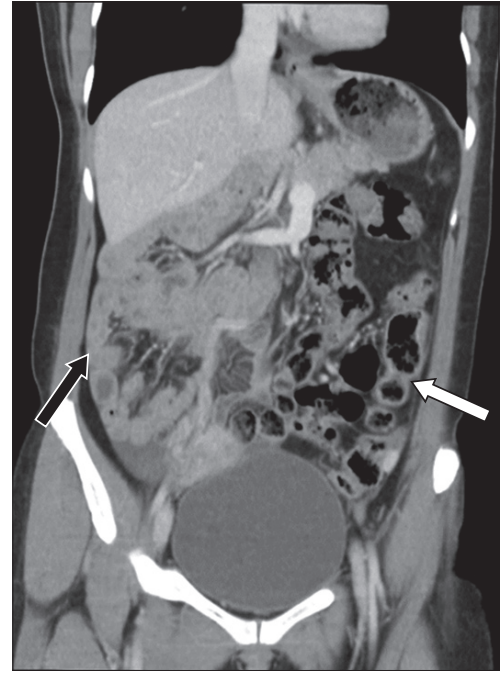

Fig. 4 A patient who was evaluated for right iliac fossa tenderness had an incidental finding of malrotation of the bowel. Coronal abdominal CT image shows the small bowel loops (black arrow) lying on the right side of the abdomen and the large bowel (white arrow) on the left. Note the reversal in position of the superior mesenteric artery and the superior mesenteric vein.
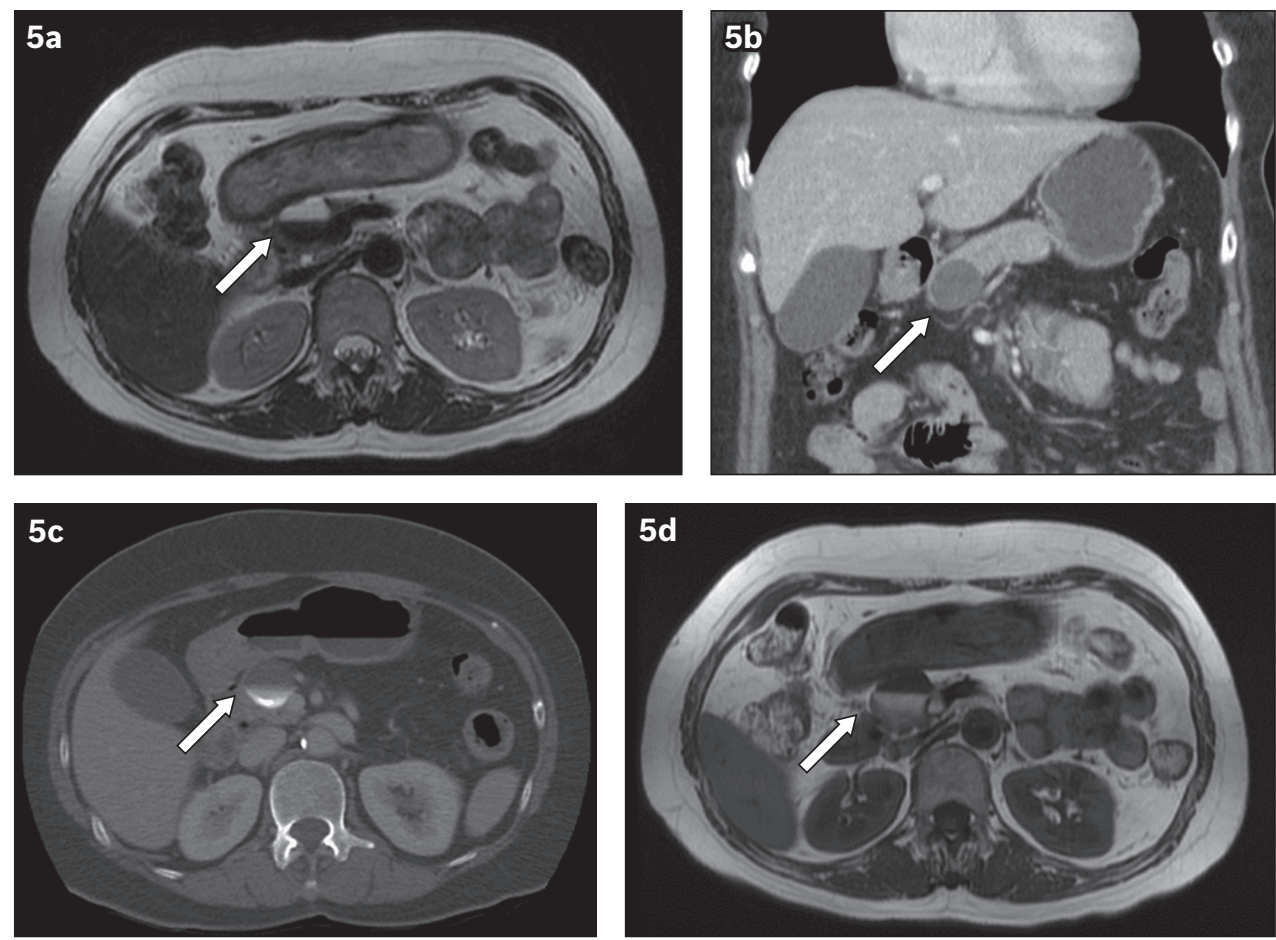

Fig. 5 (a) Axial and (b) coronal CT images, and (c) axial T2-W and (d) T1-W MR images of an elderly patient's abdomen show a smooth, thin-walled cystic structure in the head of the pancreas, with layering milk-of-calcium, compatible with a pseudocyst (arrows).

a specific magnetic resonance imaging finding that confirms the diagnosis (Fig. 5). Large pancreatic pseudocysts can cause local mass effect. ${ }^{(9)}$ They can also be complicated by secondary infection, bleeding and rupture; secondary infection is associated with a high rate of morbidity and mortality, and thus requires emergent drainage.

The normal ranges of the aortomesenteric angle and distance are debatable, although the often cited classical cut-offs are less than $25^{\circ}$ and $8 \mathrm{~mm}$, respectively. ${ }^{(3)}$ In a study of 205 abdominal CT images of paediatric patients referred for reasons other than SMAS, Arthurs et al demonstrated that $20 \%$ of patients would be diagnosed with SMAS when a cut-off aortomesenteric distance $<8 \mathrm{~mm}$ was used. ${ }^{(10)}$ Ultimately, the diagnosis of SMAS is reached using a combination of radiological and clinical features, with obstructive symptoms associated with a narrowed aortomesenteric angle and distance, as well as proximal bowel and gastric dilation.

Treatment for SMAS involves supportive measures such as the 'drip and suck' method for intravenous hydration and nasogastric tube aspiration, with strict monitoring of fluid and electrolyte status to avoid potentially fatal disturbances such as hyponatraemia. Complications such as aspiration pneumonia are 
also treated concomitantly. Thereafter, definitive management of SMAS includes a conservative weight-gain approach of enteral or parenteral feeding. ${ }^{(11)}$ Surgical management is undertaken in the following situations: (a) the patient is not amenable to a prolonged feeding strategy; (b) there is initial surgical indication (e.g. gastric perforation); or (c) conservative measures have failed. The recommended surgical procedure is duodenojejunostomy, which may be performed laparoscopically. An alternative is lysis of the ligament of Treitz with duodenal mobilisation, avoiding the need for anastomosis. ${ }^{(2,12)}$

In summary, SMAS is a rare cause of high intestinal obstruction, with multiple causative factors. It is a self-reinforcing condition, and the diagnosis is made using a combination of clinical and

ABSTRACT A 16-year-old Chinese male patient presented with constipation lasting five days, colicky abdominal pain, lethargy, weakness and body aches. He was able to pass flatus. Abdominal radiography showed a distended stomach causing inferior displacement of the transverse colon. Computed tomography revealed a dilated oesophagus, stomach and duodenum up to its third portion, with a short aortomesenteric distance and narrow angle. There was also consolidation in the lungs bilaterally. Based on the constellation of clinical and imaging findings, a diagnosis of superior mesenteric artery syndrome complicated by aspiration pneumonia was made. The patient was subsequently started on intravenous hydration, nasogastric tube aspiration and antibiotics. Following stabilisation of his acute condition, a nasojejunal feeding tube was inserted and a feeding plan was implemented to promote weight gain. The clinical presentation, differentials, diagnosis and treatment of superior mesenteric artery syndrome are discussed.

Keywords: superior mesenteric artery syndrome, upper gastrointestinal obstruction radiological features. Suggestive clinical features include relief in symptoms when the aortomesenteric angle and distance are widened in assuming a prone or left lateral decubitus position. Definitive treatment options consist of enteral or parenteral feeding and surgical measures such as duodenojejunostomy.

\section{ACKNOWLEDGEMENTS}

The authors thank Dr Hamouda Ehab Shaban Mahmoud from KK Women's and Children's Hospital for providing Figs. 3-5.

\section{REFERENCES}

1. Von Rokitansky C. Pathology for Students and Practitioners. Vienna, Austria: Bramuller \& Seidel, 1861.

2. Rudinsky SL, Matteucci MJ. Emergency department presentation of superior mesenteric artery syndrome: two cases in Marine Corps recruits. J Emerg Med 2012; 42:155-8.

3. Agrawal S, Patel H. Superior mesenteric artery syndrome. Surgery 2013; 153:601-2.

4. Ricca RL, Kasten J, Javid PJ. Superior mesenteric artery syndrome after minimally invasive correction of pectus excavatum: impact of postoperative weight loss. J Pediatr Surg 2012; 47:2137-9.

5. Merrett ND, Wilson RB, Cosman P, Biankin AV. Superior mesenteric artery syndrome: diagnosis and treatment strategies. J Gastrointest Surg 2009; 13:287-92.

6. Bergmann LL, Zimmerman P, Kaunitz JD. Unusual complication: superior mesenteric artery syndrome and closed loop gastroduodenal obstruction after adjustable gastric banding. Surg Obes Relat Dis 2011; 7:237-9.

7. Pickhardt PJ, Bhalla S. Intestinal malrotation in adolescents and adults: spectrum of clinical and imaging features. AJR Am J Roentgenol 2002; 179:1429-35.

8. Kim YH, Saini S, Sahani D, et al. Imaging diagnosis of cystic pancreatic lesions: pseudocyst versus nonpseudocyst. Radiographics 2005; 25:671-85.

9. Macari M, Finn ME, Bennett GL, et al. Differentiating pancreatic cystic neoplasms from pancreatic pseudocysts at MR imaging: value of perceived internal debris. Radiology 2009; 251:77-84.

10. Arthurs OJ, Mehta $U$, Set PA. Nutcracker and SMA syndromes: What is the normal SMA angle in children? Eur J Radiol 2012; 81:e854-61.

11. Chan DK, Mak KS, Cheah YL. Successful nutritional therapy for superior mesenteric artery syndrome. Singapore Med J 2012; 53:e233-6.

12. Mosalli R, El-Bizre B, Farooqui M, Paes B. Superior mesenteric artery syndrome: a rare cause of complete intestinal obstruction in neonates. J Pediatr Surg 2011; 46:e29-31. 


\section{SINGAPORE MEDICAL COUNCIL CATEGORY 3B CME PROGRAMME} (Code SMJ 201605B)

Question 1. Regarding superior mesenteric artery syndrome (SMAS):

a) It is a common diagnosis of upper gastrointestinal tract obstruction.

b) It is secondary to compression of the second portion of the duodenum by the superior mesenteric artery (SMA).

c) Surgical correction of scoliosis is a risk factor for its development.

d) It is a self-reinforcing condition.

Question 2. Regarding the clinical presentation of SMAS:

a) It is easily diagnosed due to its specific symptomalogy.

b) Vomiting must always be bilious due to the location of the obstruction.

c) Relief of symptoms in the prone position points to a possible diagnosis.

d) Oesophageal rupture is a known complication.

Question 3. Concerning differentials of SMAS:

a) Malrotation with midgut volvulus in adults is not possible due to its early presentation in the paediatric age group and early surgical correction.

b) Reversal of the normal right-left relationship of the superior mesenteric vein to the SMA is an imaging sign of malrotation.

c) Pancreatic pseudocyst develops 2-3 weeks after an episode of severe acute pancreatitis.

d) Secondary infection of a pseudocyst is a complication that can easily be managed conservatively.

Question 4. Concerning the diagnosis of SMAS:

a) An aortomesenteric angle of less than $25^{\circ}$ is insufficient for the diagnosis of SMAS.

b) SMAS can be diagnosed on purely clinical grounds.

c) Proximal bowel and gastric dilation to the transition point between the SMA and aorta lends support to the diagnosis of SMAS.

d) The classical normal cut-offs for the aortomesenteric angle and distance are widely acknowledged to be specific for SMAS.

Question 5. Regarding the management of SMAS:

a) Immediate parenteral nutrition is the therapy of choice.

b) Fluid and electrolyte monitoring is necessary during intravenous hydration and nasogastric tube aspiration.

c) Surgical management is the treatment of choice for all patients with SMAS.

d) Lysis of the ligament of Treitz with duodenal mobilisation is the surgical approach of choice.

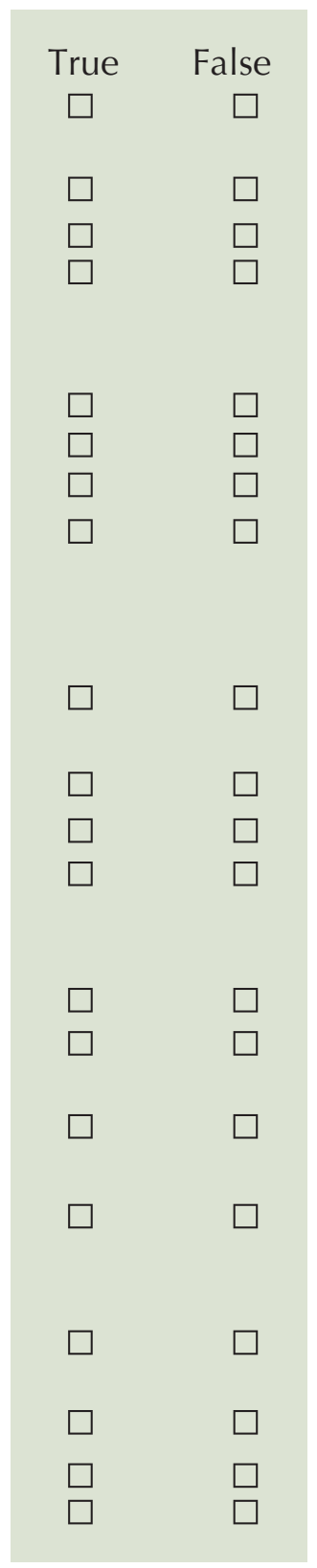

\section{Doctor's particulars:}

Name in full

MCR number

Email address

\section{SUBMISSION INSTRUCTIONS:}

(1) Log on at the SMJ website: http://www.sma.org.sg/publications/smjcurrentissue.aspx and select the appropriate set of questions. (2) Provide your name, email address and MCR number. (3) Select your answers and click "Submit".

RESULTS:

(1) Answers will be published in the SMJ July 2016 issue. (2) The MCR numbers of successful candidates will be posted online at the SMJ website by 27 June 2016. (3) Passing mark is $60 \%$. No mark will be deducted for incorrect answers. (4) The SMJ editorial office will submit the list of successful candidates to the Singapore Medical Council. (5) One CME point is awarded for successful candidates.

Deadline for submission: (May 2016 SMJ 3B CME programme): 12 noon, 20 June 2016. 